\title{
Point-of-care thrombocyte function testing using multiple-electrode aggregometry in dengue patients: an explorative study
}

Wesley de Jong ${ }^{1 *}$ (D, Tri Pudy Asmarawati ${ }^{2}$, Inge Verbeek ${ }^{1}$, Musofa Rusli ${ }^{3}$, Usman Hadi ${ }^{3}$, Eric van Gorp ${ }^{1,4}$ and Marco Goeijenbier ${ }^{1,4^{*}}$

\begin{abstract}
Background: Dengue virus (DENV) causes the hospitalisation of an estimated 500,000 people every year. Outbreaks can severely stress healthcare systems, especially in rural settings. It is difficult to discriminate patients who need to be hospitalized from those that do not. Earlier work identified thrombocyte count and subsequent function as a promising prognostic marker of DENV severity. Herein, we investigated the potential of quantitative thrombocyte function tests in those admitted in the very early phase of acute DENV infections, using Multiplate ${ }^{\text {TM }}$ multipleelectrode aggregometry to explore its potential in triage.

Methods: In this prospective cohort study all patients aged $\geq 13$ admitted to Universitas Airlangga Hospital in Surabaya, Indonesia with a fever $\left(\geq 38^{\circ} \mathrm{C}\right.$ ) between 25 January and 1 August 2018 and with a clinical suspicion of DENV, were eligible for inclusion. Exclusion criteria were a thrombocyte count below $100 \times 109 / \mathrm{L}$ and the use of any medication with a known anticoagulant effect, nonsteroidal anti-inflammatory drugs and acetyl salicylic acid. Clinical data was collected and blood was taken on admission, day 1 and day 7. Samples were tested for acute DENV, using Panbio NS1 ELISA. Platelet aggregation using ADP-, TRAP- and COL-test were presented as Area Under the aggregation Curve (AUC). Significance was tested between DENV+, probably DENV, fever of another origin, and healthy controls $(\mathrm{HC})$.

Results: A total of 59 patients (DENV $n=10$, DENV probable $n=25$, fever other origin $n=24$ ) and $20 \mathrm{HC}$ were included. We found a significantly lower thrombocyte aggregation in the DENV+ group, compared with both HCs and the fever of another origin group $(p<.001)$. Low ADP AUC values on baseline correlated to a longer hospital stay in DENV+ and probable DENV cases.

Conclusion: Thrombocyte aggregation induced by Adenosine diphosphate, Collagen and Thrombin receptor activating peptide-6 is impaired in human DENV cases, compared with healthy controls and other causes of fever. This explorative study provides insights to thrombocyte function in DENV patients and could potentially serve as a future marker in DENV disease.
\end{abstract}

Keywords: Dengue, Multiplate, Haemostasis

\footnotetext{
* Correspondence: w.dejong.2@erasmusmc.nl; m.goeijenbier@erasmusmc.nl

'Department of Viroscience, Erasmus MC, Rotterdam, the Netherlands

Full list of author information is available at the end of the article
} 


\section{Introduction}

Dengue virus (DENV), most likely the most important mosquito transmitted viral disease in the world, is endemic in South East Asia. The estimated number of clinical infections worldwide is 67-136 million [1], with an estimated 500,000 people requiring hospitalisation every year. For Indonesia, which has a population of over 260 million people [2], DENV accounts for up to $55 \%$ of febrile cases for which a visit to a healthcare professional is necessary and in which there is a potential need of hospitalisation [3]. Upon infection, patients can present symptoms ranging from a simple flu-like illness up to severe disease warranting hospitalisation due to shock and/or haemorrhage. No treatment, nor an effective vaccine is available for DENV, which means that those who develop a severe form of the disease rely on supportive care, mainly consisting of the maintenance of the bodyfluid volume [4]. In densely populated areas of Indonesia, such as the Java Island, DENV outbreaks can severely stress the capacity of healthcare systems, due to the large number of persons simultaneously seeking medical care $[5,6]$. Earlier studies tried to identify patients at risk of developing severe dengue by specific biomarkers, genomics, machine learning and early point-ofcare ultrasound. However, many of these promising markers call for difficult, expensive or laborious techniques that are not practical in the current Indonesian healthcare setting [7-10]. For adequate DENV diagnosis, clinicians should rely on serological (IgM/IgG) and/or NS1 detection or molecular tools. However, these assays are not routinely available in outbreak areas or come with a certain delay. Currently, it is difficult to early differentiate uncomplicated dengue from those that will develop a severe form of the disease that warrants intensified in-hospital monitoring. Such approaches were previously studied in a specific combination of hematological parameters to differentiate between dengue and malaria in Thailand [11].

Excellent studies have explored the role of thrombocytes in viral infections [12-14]. Thrombocytes not only play a crucial role in primary haemostasis, they are also known to play an important role in inflammatory responses, host defense and vascular integrity $[15,16]$. Thrombocytopenia is commonly observed in patient with a DENV infection. The magnitude of thrombocytopenia, or "drop" in platelets seems to strongly correlate with DENV severity. Thrombocytopenia in DENV is mainly the result of decreased platelet production in the marrow and increased platelet destruction [17]. Interestingly, bleeding also occurs in DENV patients with thrombocyte counts within normal range, suggesting there is an important role for alterations in thrombocyte function or activation [18]. For thrombocyte activation, this leads to deposition of aggregates in microvascular structures. In this context, a number of surface markers such as P-selectin and
CD63 expression, have been studied and these correlate to a decline in thrombocyte counts. Thrombocyte activation was found to be at a maximum after DENV clearance, indicating the presence of continuing thrombocyte activation mechanisms in the convalescent phase of the disease $[12,15]$.

Clinical studies of hospitalised DENV patients showed correlations between the occurrence of bleeding and thrombocytopenia and the need for a prolonged hospital stay [19]. During this early clinical phase, impaired thrombocyte function is not detected in routinely tests, but altered aggregation might relate to duration of hospital stay. To test this hypothesis, bench-top tests to assess thrombocyte function that are characterized by a short turn-around time would be suitable. In this matter, point-of-care thrombocyte aggregation tests are extensively used in the field of cardiology and cardiothoracic surgery [20], while a small number of studies are looking at platelet function in neurology [21], gynaecology [22] and sepsis [23]. Furthermore, patients presenting with Haemorrhagic Fever and Renal Syndrome (HFRS) due to Puumala orthohantavirus infection, showed impaired thrombocyte aggregation on almost all test reagents when bedside platelet aggregation was assessed using Multiplate $^{\circledast}$ [24]. The Multiplate ${ }^{\bullet}$ analysis is based on the principle that platelets become sticky upon activation by reagents and adhere. These aggregate onto metal sensor wires in the Multiplate ${ }^{\circledR}$ analyser, which then measures an increase in electrical resistance. Over a six-minute timeframe, aggregation units (AU) are plotted against time, resulting in an Area Under the aggregation Curve (AUC), in Units. Adenosine diphosphate (ADP) is an important general agonist for platelet aggregation [25]. Collagen mediates the integrity of the vascular wall and its actions prevent excessive hemorrhage or thrombosis [26]. Last, thrombin receptor activator for peptide 6 (TRAP-6) acts via thrombin receptor protease-activating receptor-1, which is highly expressed on platelets [27].

Clinicians in low- to middle income countries where DENV is endemic, usually rely on the WHO 2009 case description to estimate the clinical course of a patient $[28,29]$. If locally available, additional rapid DENV nonstructural protein 1 (NS1) tests play a limited role in the decision-making process, especially taking into account that some patients present quite late to the hospital, when the virus was already neutralised and hence no NS1 is present. ELISA-based testing is known to have better characteristics, but it is more laborious and is only suitable for batch testing. As stated previously, additional understanding of thrombocyte function in DENV infections would be of value for healthcare settings in Indonesia, or indeed elsewhere.

In this study we explore the use Multiplate ${ }^{\mathrm{Tm}}$ multipleelectrode aggregometry to evaluate its potential as marker of DENV severity expressed by the duration of 
hospital stay in the very early phase of DENV infectionin Indonesia.

\section{Methods \\ Patients and controls}

Consecutive patients were included in the study, which ran from 25 January to 1 Augustus 2018 at the department of internal medicine of the Universitas Airlangga hospital in Surabaya, East-Java, Indonesia. Patients aged 13 or older, presenting with a fever $\left(\geq 38^{\circ} \mathrm{C}\right)$ on the ward or Emergency Department, and for whom there was a clinical suspicion of DENV infection in accordance with the case definitions in the WHO Dengue 2009 guideline [28], were deemed eligible for inclusion. Exclusion criteria were a thrombocyte count below $100 \times 10^{9}$ / $\mathrm{L}$ and the use of any medication with a anticoagulant and/or antiplatelet effect, including nonsteroidal antiinflammatory drugs (NSAIDs), acetyl salicylic acid (ASA), non-vitamin $\mathrm{K}$ oral anticoagulants (NOAC), direct oral anticoagulants (DOAC) and antiplatelet drugs such as clopidogrel, dipyridamole or vitamin $\mathrm{K}$ antagonists. Also, patients with inherited platelet function disorders (e.g. Von Willebrand disease) were excluded. After obtaining written informed consent from patients or their legally authorised representatives, we collected blood samples on baseline, day 1 and $7(+/-48 \mathrm{~h})$. Patients were followed up according to local practices and guidelines. Based on the clinician's discretion, this included imaging, urinalysis and blood culture.

\section{Sample collection}

All samples were drawn using a BD vacutainer system using $0.7 \mathrm{~mm} \times 25 \mathrm{~mm}$ needles, and tubes were gently inverted five times after blood was drawn. Multiplate samples were drawn to Diapharma Multiplate ${ }^{\bullet}$ Hirudin Blood Tubes. At inclusion, an additional BD Vacutainer ${ }^{\circ}$ $\mathrm{SST}^{\mathrm{Tm}}$ serum tube was taken and spun down in a centrifuge at $2000 \mathrm{RCF}$ (room temperature) for $15 \mathrm{~min}$.

\section{Dengue virus diagnostics and study group assignment}

Panbio $^{\circ}$ Dengue Early Rapid test was used to identify dengue cases by ascertaining the presence of the NS1antigen in the serum. This information was used to track the number of patients in each group throughout the study. Residual serum was aliquoted, and stored at minus $80^{\circ} \mathrm{C}$ while awaiting further processing. Stored serum samples were tested at a later stage with a Panbio ${ }^{\circ}$ Dengue Early ELISA dengue NS1 antigen capture ELISA, according to the manufacturer's specifications. In case of a positive NS1 ELISA, patients were assigned to the study group DENV-confirmed. Consequently, patients were assigned to "fever of another origin" in case of a clear working diagnose other than DENV. Others were evaluated against WHO 2009 guidelines [28] and classified in the DENV probable group. Full criteria are available from Appendix A. For logistical reasons, no convalescent samples could be collected for IgM/IgG testing.

\section{Platelet aggregation measurement using multiplate}

Using Multiplate, Hirudin blood tubes were tested using ADP and TRAP- 6 reagents, as per the manufacturer's instructions and COL reagent. In brief: ADP and TRAP6 reagents were reconstituted with $1000 \mu \mathrm{L}$ high purity distilled water and aliquoted in vials of $110 \mu \mathrm{L}$. These reagents were stored at minus $80^{\circ} \mathrm{C}$ for a maximum of 4 weeks, then while they were in use kept at between 2 and $8{ }^{\circ} \mathrm{C}$ for a maximum of 7 days. COL reagent (CHRONO-LOG ${ }^{\circ}$ corporation, $\mathrm{P} / \mathrm{N}$ 385) was prepared by mixing $50 \mu \mathrm{L} \mathrm{COL}$ with $450 \mu \mathrm{L}$ isotonic glucose solution ( $\mathrm{pH}$ 2.7-2.9). The prepared COL reagent was used for a maximum of 1 week and kept at between 2 and $8{ }^{\circ} \mathrm{C}$. Using $20 \mu \mathrm{l}$ of the solution in a Multiplate test cell, the final concentration achieved was $3.2 \mu \mathrm{g} / \mathrm{ml}$. Blood testing in the Multiplate was done a maximum of $4 \mathrm{~h}$ after blood draw. To start the Multiplate measurements, $300 \mu \mathrm{L}$ of sodium chloride $0.9 \%$ solution was mixed with $300 \mu \mathrm{L}$ of Hirudinized whole blood at $37^{\circ} \mathrm{C}$ for $3 \mathrm{~min}$, after which $20 \mu \mathrm{L}$ of the reagent (either ADP, TRAP or $\mathrm{COL}$ ) was added. For the statistical analysis, tests were performed in duplo, using the mean of two similar tests (i.e. same patient/reagent). Due to logistical reasons, not all samples that were available could be tested with COL reagent (see the results).

\section{Statistical analysis}

Based on earlier work by Peerschke and colleagues, to detect a hypothesised AUC 20 difference in baseline means between study groups with a power of $80 \%$, we calculated the following sample sizes: ADP $n=27$, TRAP $n=20$ and COL $n=30$ respectively [30]. Statistical analysis was performed using IBM SPSS statistics software (for Windows, version 25). Normality testing was performed using Shapiro-Wilk (with a value of .05 or above considered as normally distributed data). If the data was distributed normally, the means were compared with a t-test, or else using a Kruskal-Wallis and Mann-Whitney U-test. Ethical clearance was obtained from the ethical committee of the Universitas Airlangga hospital, with reference $126 / \mathrm{KEH} / 2017$, and all study procedures were conducted following the Declaration of Helsinki [31].

\section{Results}

\section{Study-group assignment}

We enrolled 60 subjects; all of them were laboratory tested for the presence of DENV NS1 antigen in serum. Figure 1. shows an overview of the enrolment of the participating patients, who were subsequently assigned to 


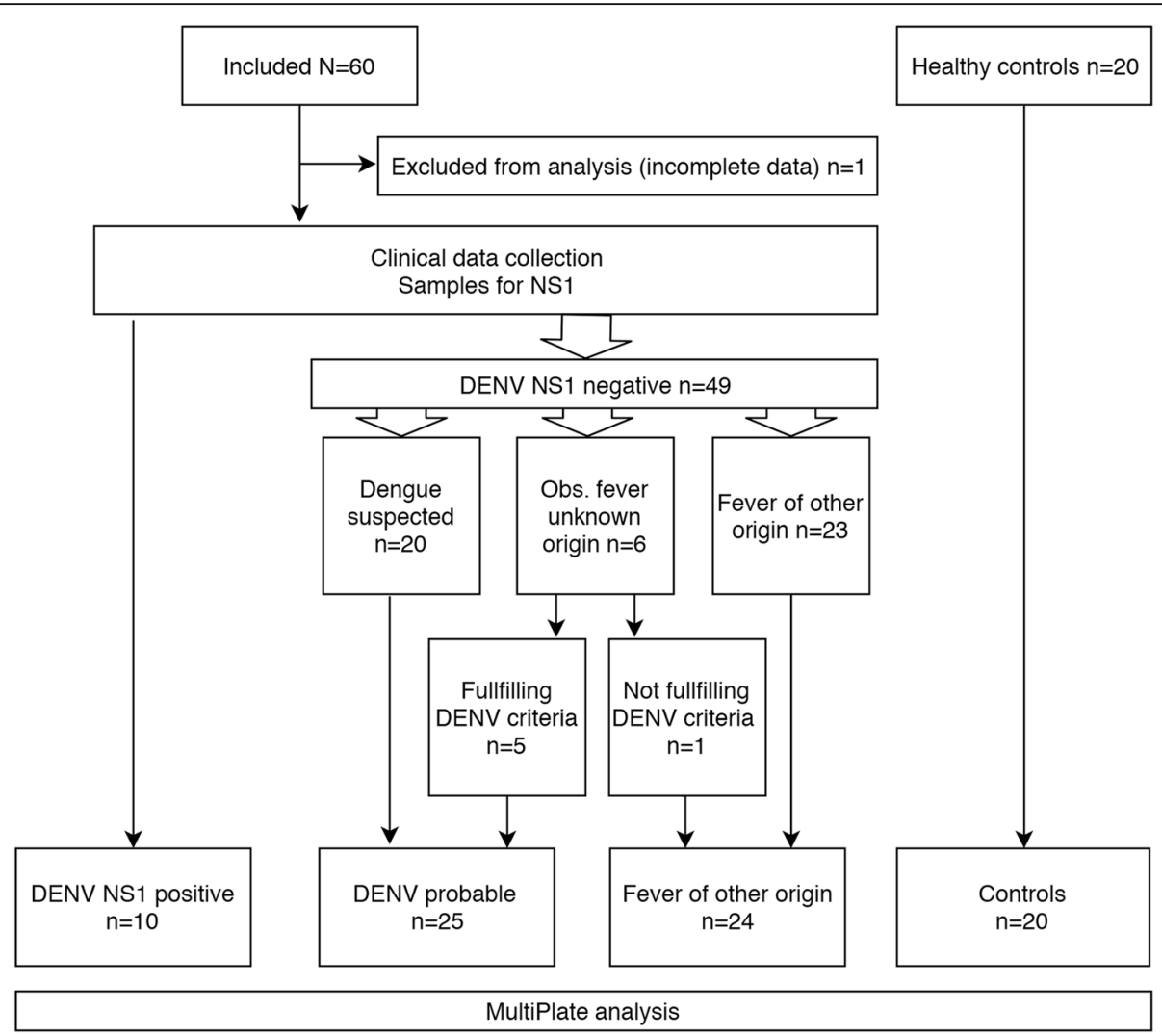

Fig. 1 Flowchart for the analysis of baseline (day of inclusion) samples following the CONSORT 2010 Statement ${ }^{1}$. The data shown for DENV NS1 positive is based on the DENV NS1 ELISA results (see methods, Appendix A and Supplementary Table 1.). DENV criteria following WHO 2009 Dengue guidelines. 1| Schulz KF, Altman DG, Moher D, for the CONSORT Group. CONSORT 2010 Statement: updated guidelines for reporting parallel group randomised trials. BMC Medicine 2010, 8:18

the different groups. During the study period, 9 patients initially tested positive using the DENV NS1 rapid test, while 1 additional DENV case was later confirmed by using the more sensitive DENV NS1 ELISA (totalling $17 \%$ of all the subjects, see Supplementary Table 1.). We assigned 59 patients to the following study groups: DENV-confirmed $(n=10)$, DENV-probable $(n=25)$ and fever of another origin $(n=24)$. For reasons of incomplete data, 1 patient was excluded from the analysis. A group of healthy hospital workers served as the control population $(n=20)$.

\section{Baseline study and disease characteristics}

Baseline and clinical characteristics are shown in Table 1. With a mean age of 19, the DENV-confirmed group was significantly younger than that of the fever of another origin group (mean age 28), $\mathrm{U}=34, p=.001$ ). The mean, self-reported fever duration was 3.60 days for DENVconfirmed cases, 4.64 for DENV-probable $(\mathrm{U}=70, p=$ $.039)$ and there was a mean duration of 6.63 days for those with a fever of another origin $(U=67, p=.043)$. Subjects classified as having a fever of another origin presented with complaints suspected for: a respiratory-tract infection
( $n=5,20 \%)$, typhoid fever $(n=9,38 \%)$, a urinary-tract infection $(n=2,8 \%)$, other abdominal focus $(n=4,17 \%)$ and undifferentiated fever/sepsis $(n=4,17 \%)$. We observed the presence of DENV signs and symptoms in all study groups. Leukocyte counts for DENV-confirmed cases were significantly lower compared with both DENVprobable (a mean of $3.24 \times 10^{9} / \mathrm{L}$ vs $5.56 \times 10^{9} / \mathrm{L}(\mathrm{U}=46$ $p=.004)$ ), as well as a fever of another origin (a mean of $\left.10.72 \times 10^{9} / \mathrm{L} \mathrm{U}=11 p<.001\right)$. As shown in Fig. 2 ., the mean thrombocyte count for DENV-confirmed cases was $156 \times 10^{9} / \mathrm{L}$, while for DENV-probable cases it was $150 \times$ $10^{9} / \mathrm{L}$ and for a fever of another origin it was $284 \times 10^{9} / \mathrm{L}$ $(\mathrm{U}=56, p=.016$ for DENV-confirmed vs a fever of another origin). No subjects showed signs of shock on enrolment, as the mean arterial pressure (MAP) was $\geq 65$ $\mathrm{mmHg}$ for all. The mean duration of hospital stay, following the clinicians discretion, for all subjects was 2.05 days, with a range of 0 to 8 days and no significant differences between groups (DENV-confirmed vs DENV-probable $p=.051 \mathrm{U}=74$, DENV-confirmed vs Other origin $p=$ $0.152 \mathrm{U}=83$ ). While not significant, it should be noted that the mean hospital stay duration for the DENVconfirmed group, i.e. those that tested NS1 positive on 
Table 1 Baseline study characteristics

\begin{tabular}{|c|c|c|c|c|c|c|}
\hline & $\begin{array}{l}\text { Study population } \\
\boldsymbol{N}=59\end{array}$ & $\begin{array}{l}\text { Dengue NS1 } \\
\text { confirmed } \boldsymbol{n}=10\end{array}$ & $\begin{array}{l}\text { Dengue probable } \\
\boldsymbol{n}=25\end{array}$ & $\begin{array}{l}\text { Fever of another origin } \\
\boldsymbol{n}=24\end{array}$ & $\begin{array}{l}\text { Control group } \\
\mathbf{N}=20\end{array}$ & $\begin{array}{l}\boldsymbol{p} \text { - } \\
\text { value }\end{array}$ \\
\hline Mean age (range) & $28(14-71)$ & $19(14-36)$ & $24(14-54)$ & $35(14-71)$ & $30(21-46)$ & $<.05^{*}$ \\
\hline Male sex $n(\%)$ & $30(50.8 \%)$ & $3(30 \%)$ & $16(64 \%)$ & $11(45.8 \%)$ & $9(45 \%)$ & .271 \\
\hline Duration of fever days (range) & $5.27(1-30)$ & $3.6(2-4)$ & 4.6 & $6.63(1-30)$ & N/D & .058 \\
\hline Headache $n(\%)$ & 39 (66.1\%) & $9(90 \%)$ & $19(75 \%)$ & $11(45.8 \%)$ & N/D & $.018^{\circ}$ \\
\hline Retro-orbital pain & $4(6.8 \%)$ & $0(0 \%)$ & $4(16 \%)$ & $0(0 \%)$ & N/D & .054 \\
\hline Nausea, vomiting & $50(84.7 \%)$ & $8(80 \%)$ & $22(88 \%)$ & $20(83 \%)$ & N/D & .812 \\
\hline Rash & $3(5.1 \%)$ & $1(10 \%)$ & $1(4 \%)$ & $1(4.2 \%)$ & N/D & .740 \\
\hline Swollen glands & $2(3.3 \%)$ & $1(10 \%)$ & $0(0 \%)$ & $1(4.2 \%)$ & N/D & .324 \\
\hline Aches and pains & $18(30.5 \%)$ & $2(20 \%)$ & $9(36 \%)$ & $7(29.2 \%)$ & N/D & .639 \\
\hline Mean MAP ${ }^{\circ}$ (range) & $90(67-125)$ & $89(76-107)$ & $97(67-121)$ & $92(69-125)$ & N/D & .689 \\
\hline Mean temperature (range) & $38.3(36.0-40.0)$ & $38.3(36.8-39.4)$ & $38.4(36.6-39.7)$ & $38.3(36.0-40.0)$ & N/D & .962 \\
\hline Mean leukocyte count (range) & $7.27(1.24-22.94)$ & $3.24(1.24-6.17)$ & $5.56(1.88-8.90)$ & $10.7(3.72-22.94)$ & N/D & $<.05^{*}$ \\
\hline $\begin{array}{l}\text { Mean thrombocyte count } \\
\text { (range) }\end{array}$ & $205(101-815)$ & $156(101-196)$ & $150(111-210)$ & $284(123-815)$ & N/D & $<.05^{*}$ \\
\hline Leukocytosis & $13(22.0 \%)$ & $0(0 \%)$ & $0(0 \%)$ & $13(54.2 \%)$ & N/D & $<.05^{*}$ \\
\hline Leucopenia & $13(22.0 \%)$ & $8(80 \%)$ & $5(20 \%)$ & $0(0 \%)$ & N/D & $<.05^{*}$ \\
\hline Thrombocytosis & $4(6.8 \%)$ & $0(0 \%)$ & $0(0 \%)$ & $4(16.7 \%)$ & N/D & $.044^{*}$ \\
\hline Thrombopenia & $24(40.7 \%)$ & $4(40 \%)$ & $14(56 \%)$ & $6(25.0 \%)$ & N/A & .087 \\
\hline Increased creatinine ${ }^{+}$n/N (\%) & 2/38 (5.2\%) & 0/6 (0\%) & $2 / 21(9.5 \%)$ & $0 / 11(0 \%)$ & N/D & $\mathrm{N} / \mathrm{C}$ \\
\hline $\begin{array}{l}\text { X-ray suspected pneumonia n/ } \\
\text { N (\%) }\end{array}$ & $3 / 15$ & N/D & $0 / 3(0 \%)$ & $3 / 12(25 \%)$ & N/D & $\mathrm{N} / \mathrm{C}$ \\
\hline $\begin{array}{l}\text { Urine dipstick/culture positive } \\
\text { n/N (\%) }\end{array}$ & 7/11 (63\%) & $1 / 1(100 \%)$ & $0 / 4(0 \%)$ & 6/6 (100\%) & N/D & $\mathrm{N} / \mathrm{C}$ \\
\hline Blood culture positive n/N (\%) & $1 / 1$ & N/D & N/D & 1/1 (100\%) & N/D & $\mathrm{N} / \mathrm{C}$ \\
\hline $\begin{array}{l}\text { Duration of hospital stay in } \\
\text { days (range) }\end{array}$ & $2.05(0-8)$ & $2.50(1-4)$ & $1.80(1-4)$ & $2.13(0-8)$ & N/D & $\mathrm{N} / \mathrm{C}$ \\
\hline
\end{tabular}

Patients with fever on admission were scored for anamnestic parameters according to the WHO dengue 2009 guideline. Cases that were not NS1-confirmed were considered probable dengue on condition that at least two of the following symptoms were presented: headache, retro-orbital pain, muscular/joint pain, nausea/ vomiting, swollen glands, rash, leukopenia. Continuous and semi-continuous data was analysed using the Kruskal-Wallis test and categorical data was analysed using the Chi-Squared test. Leukopenia defined as leukocyte count $<3.6 \times 10^{9} / \mathrm{L}$; Leukocytosis defined as leukocyte count $>11.0 \times 10^{9} / \mathrm{L}$; Thrombocytopenia defined as thrombocyte count $<150 \times 10^{9} / \mathrm{L}$; Thrombocytosis defined as thrombocyte count $>400 \times 10^{9} / \mathrm{L} \mathrm{N} / \mathrm{D}$ not done; N/C not calculated (for limited data); In case of incomplete data, data is shown as number/Number evaluated $(\mathrm{n} / \mathrm{N}){ }^{*}{ }^{*}$ significant $(p<.05){ }^{\circ}$ Mean Arterial Pressure; ${ }^{+}$incomplete data for 21 cases

baseline, was 2.5 days, as opposed to 1.8 for the DENVprobable group - which is reflected by a decline in numbers analysed as shown in Fig. 3. One subject died (cause unknown, from the fever of another origin group) and one subject was referred for third-line treatment in another hospital (reason unknown).

\section{MultiPlate analysis}

As shown in Fig. 3, MultiPlate analysis was available for ADP, COL and TRAP reagents in 59 (100\%), 47 (80\%), and $59(100 \%)$ of the subjects, respectively, at baseline. The Area Under the aggregation Curve (AUC, in Units or $\mathrm{U}$ ) for ADP, TRAP and COL on baseline was significantly lower for both DENV-confirmed vs Healthy controls $(p<.001$ for all analyses) and for DENV-confirmed vs fever of another origin ( $\mathrm{p}<.001$ for all analyses).
Meanwhile, Table 2. shows the relationship between baseline ADP, TRAP and COL measurements and the final duration of hospital stay. Firstly, using Spearman's rank-order correlation, we found no correlation for ADP, TRAP, COL measurements at baseline for DENVconfirmed versus length of stay (ADP: $\operatorname{rs}(10)=-.065$., $p=.858$; TRAP: $\operatorname{rs}(10)=-. .241, p=.503$; COL: $\operatorname{rs}(10)=$ $.085, p=.816)$ or for DENV-probable versus length of stay $\left(A D P: r_{s}(25)=-.371\right.$., $p=.068 ;$ TRAP: $r_{s}(25)=$ $-.299, p=.147$; COL $\left.r_{s}(18)=-.296, p=.232\right)$. Secondly, however, given that it was considered highly likely that subjects in the DENV-probable group indeed had a DENV infection and were clinically followed-up as such, we merged both DENV-confirmed and DENV-probable groups. We found that a lower ADP AUC correlated to a hospital stay of $>1$ day (ADP: $r_{s}(35)=-.360, p=.033$ ). 


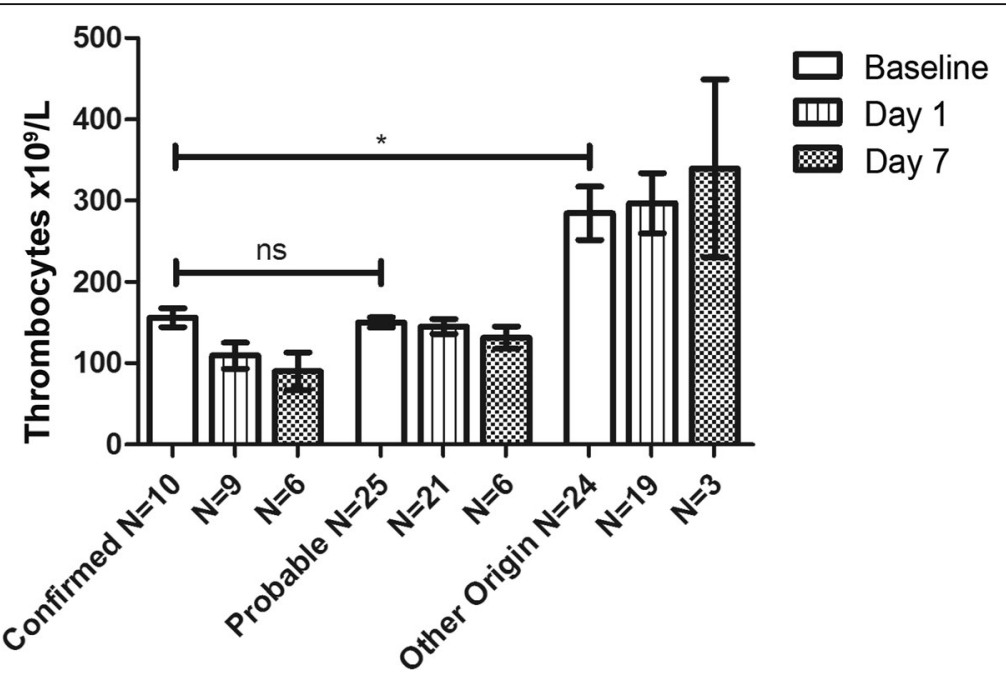

Fig. 2 Thrombocyte counts $\left(\times 10^{9} / \mathrm{L}\right)$ presented as Mean/Standard Error of the Mean (SEM) for baseline (day of inclusion), day 1 , day 7 (+/ $-48 \mathrm{~h}$ or at discharge). $N$ = represents number of samples available. Data for Confirmed and Other Origin groups is not normally distributed. No data available for healthy controls. Statistical analysis using Mann-Whitney U-test. * = significant $p=.0173$; ns = not significant

For TRAP and COL, this effect was not observed (TRAP: $\mathrm{r}_{\mathrm{s}}(35)=-, 321, p=.060 ;$ COL $\left._{\mathrm{s}}(28)=-.305, p=.115\right)$.

\section{Validity of MultiPlate results versus thrombocyte counts}

The internal validity of results reported in the MultiPlate analysis depends on the reported thrombocyte counts (see discussion). Moreover, as mentioned earlier, compared with the fever of another origin group, thrombocyte counts were significantly lower in the DENVconfirmed group. Furthermore, the same holds true for ADP, TRAP and COL AUC. Using Spearman's correlation we checked whether thrombocyte counts at baseline correlated to ADP AUC at baseline. While we found no correlation for DENV-confirmed and DENVprobable $\mathrm{rs}(35)=.231, p=.181$, we did find a significant positive correlation for the fever of another origin, $\operatorname{rs}(24)=.765, p<.001$.

\section{Clinical implementation}

With the data reported in the previous paragraphs, we could potentially provide additional decision-making rules based on information that is readily available to the treating physician. First of all, a binary linear regression analysis was run to calculate odds-ratios for assignment to the DENV-positive/DENV-probable group, based on ADP values on baseline, the presence of leukocytopenia $\left(<3.6 \times 10^{9} / \mathrm{L}\right)$ and the presence of thrombocytopenia $(<$ $150 \times 10^{9} / \mathrm{L}$ ). For this analysis, cut-off values for ADP AUC baseline of $<40,<35$ and $<30$ were chosen. As can be deducted from Fig. 3., as well as the calculations mentioned earlier, in the current study this range of ADP AUC values would seem to be specific to DENV patients. DENV-confirmed/DENV-probable could be predicted from the whole study population $(72.9 \%$ correct) by using an ADP cut-off of $<40(\beta=-2.189 p=$ $.003)$ and establishing the presence of leukocytopenia $(\beta=20.410 p=.998)$ and thrombocytopenia $(\beta=.384 p=$ .611 ). If there is no leukocytosis (i.e. leukocyte count < $11.0 \times 10^{9} / \mathrm{L}$ ), the overall percentage of correct studygroup prediction in the model increases to $83.1 \%$, with the following characteristics: ADP cut-off of $<40(\beta=-$ $2.383 \mathrm{p}=.003)$, leukocyte count $<11 \times 10^{9} / \mathrm{L}(\beta=21.878$ $\mathrm{p}=.998$ ), thrombocytopenia (and leukocytopenia or normal leukocyte range $(\beta=22.18, \mathrm{p}=.998)$, constant $(\beta=-$ $17.206 p=.999)$. Secondly, to further simplify the model, we found that presenting with a headache as a clinical parameter with ADP AUC values could predict $79.7 \%$ of the cases (ADP $<40 \beta=-2.669 p<0.001$, headache $\beta=$ $1.459 p<0.043$, constant $\beta=3.429 p<0.003)$.

\section{Trends in MultiPlate values on follow-up}

Additionally, Fig. 3. also shows ADP (3a), COL (3b) and TRAP (3c) values during follow-up at day 1 and day 7 $(+/-48 \mathrm{~h})$. Due to the discharge of subjects, the number of available follow-up samples is limited. In the fever of another origin group, AUC values for ADP, COL and TRAP are stable (not significantly different) during follow-up. For the DENV-probable group, a significant recovery of ADP is observed from baseline to day 1 follow-up (a mean ADP AUC of 34.85 at baseline and 43.37 at day 1 follow-up respectively; $p=.009$ using paired samples t-test). For COL and TRAP this was not the case (tested with paired samples t-test for all time points and study groups). 


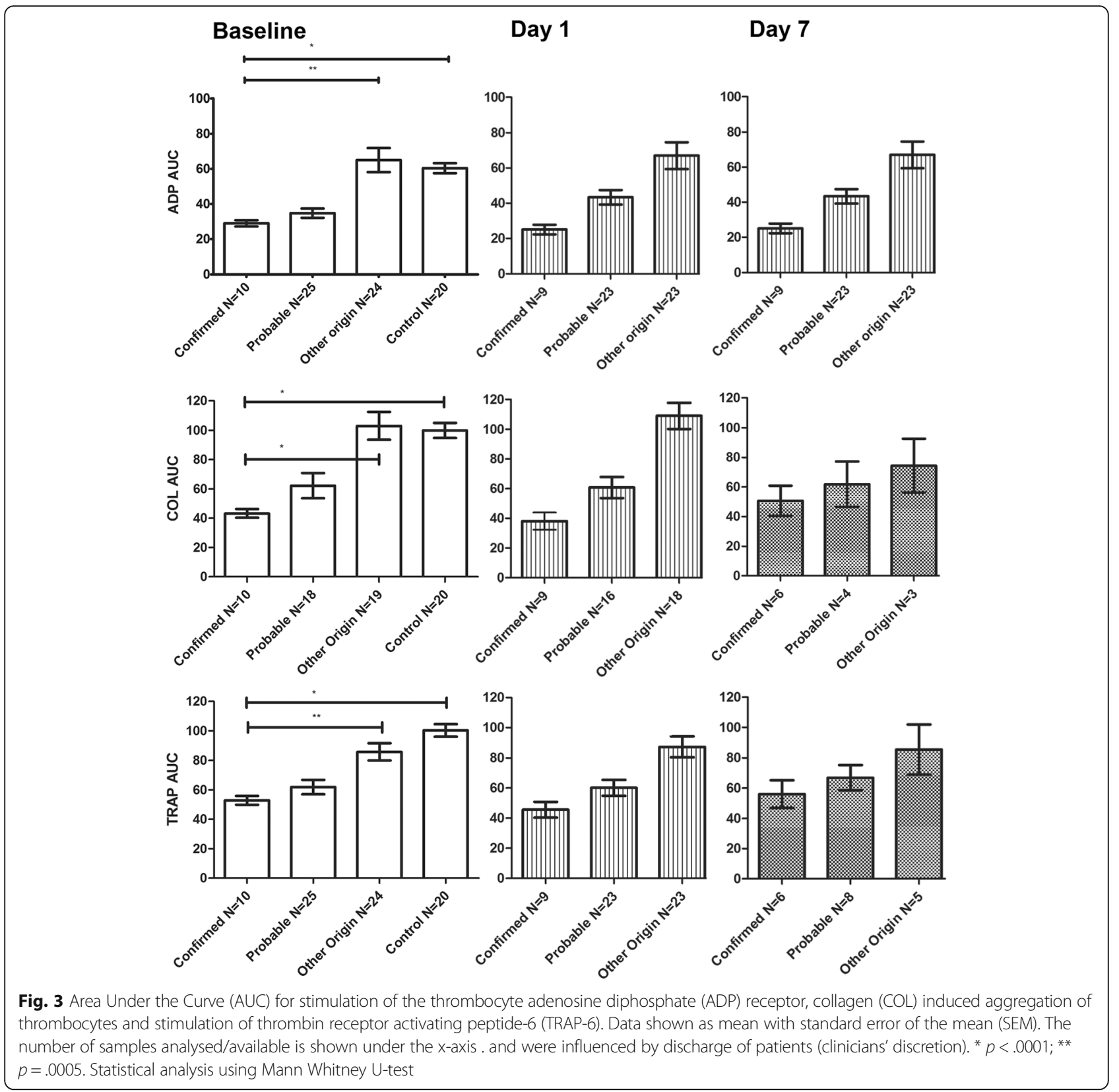

Table 2 Predictive value of low ADP on admission for hospital stay $>1$ day

\begin{tabular}{|c|c|c|c|c|c|c|}
\hline & \multicolumn{2}{|c|}{ Hospital stay $<=1$ day or above } & \multirow{2}{*}{$\begin{array}{l}\boldsymbol{p}- \\
\text { value }\end{array}$} & \multicolumn{2}{|c|}{ Hospital stay $<=2$ days or above } & \multirow{2}{*}{$\begin{array}{l}\boldsymbol{p} \text { - } \\
\text { value }\end{array}$} \\
\hline & Stay $<=1$ day & Stay $>1$ day & & Stay $<=2$ days & Stay $>2$ days & \\
\hline ADP $n$ & 12 & 23 & & 26 & 9 & \\
\hline ADP mean (IQR) & $40.25(29.25)$ & $29.39(10.50)$ & $.008^{* *}$ & $35.54(15.63)$ & $26.11(10.00)$ & $.056^{*}$ \\
\hline TRAP n & 12 & 23 & & 26 & 9 & \\
\hline TRAP mean (IQR) & $66.54(37.38)$ & $55.63(23.00)$ & $.123^{*}$ & $62.84(25.00)$ & $49.33(14.75)$ & $.055^{* *}$ \\
\hline COL n & 10 & 18 & & 20 & 8 & \\
\hline COL mean (IQR) & $65.47(37.00)$ & $49.94(19.50)$ & $.099^{*}$ & $59.66(32.25)$ & $45.06(14.25)$ & $.500^{*}$ \\
\hline
\end{tabular}

Comparison of mean baseline (T1) Area Under the Curve values for ADP, TRAP and COL for patients with either confirmed dengue or probable dengue (grouped together, $n=35$ ) to the length of hospital stay. ${ }^{*}$ P-values calculated using Mann-Whitney U test. ${ }^{* *}$ Normally distributed data (Shapiro-Wilk $>.05$ ), statistical analysis using independent samples t-test 


\section{Discussion}

We assessed thrombocyte aggregation in acute DENV cases using multiple-electrode aggregometry (MultiPlate). Our data suggests that UAC values of ADP are not only significantly lower in acute DENV cases, but that they might also be associated with a prolonged hospital stay and could therefore support the estimation of disease severity. To the best of our knowledge this is the first study that has applied short-turnaround time thrombocyte aggregation tests in acute DENV cases. This explorative data contributes to the understanding of thrombopathies as result of a DENV infection and we suggest that thrombocyte aggregation tests, such as the MultiPlate, could be further studied in the clinical assessment of DENV-suspected patients.

The presence of DENV non-structural protein 1 (NS1) antigen in blood is known to relate to the early stages of DENV [32]. Subjects classified as "DENV-probable" in the current study, comply with the WHO DENV case description [28], but tested negative for NS1. This could be explained by the potential neutralisation of the NS1 antigen in re-infected patients in a highly endemic area, the diagnostic inaccuracy of the available tests, or a delayed clinical presentation - as subjects classified as "DENV-probable" had a longer self-reported duration of fever, compared to the "DENVconfirmed" subjects. Diagnostic methods with high sensitivity and specificity for the detection of DENV NS1, including reverse transcriptase PCR (RT-PCR), were not available here [33] and no convalescent samples with sufficient timeintervals (i.e. to detect fold rise in antibody titers) were collected for logistical reasons. The alternatively used enzymelinked immunosorbent assay (ELISA) for DENV NS1 detection [34] marginally outperformed the NS1 rapid testing in our study. In future, point-of-care approaches with a lower limited of detection, might find its way into resource limited settings, and could be used instead [35]. Circulating DENV serotypes that the authors were not aware of during the study period might have affected the accuracy and overall result found, because, as was previously shown, compared with other serotypes the sensitivity of detecting NS1 for DENV-2 and DENV-4 serotypes, in particular, is somewhat limited [36]. This might explain why the number of confirmed DENV cases doesn't correspond with other studies. These report a higher number of confirmed DENV (albeit, using more extensive diagnostic assays) in similar settings of up to $72 \%$ [37, 38]. Furthermore, according to recent data from Surabaya, in studies conducted by Wardhani et al., up to $66 \%$ of adults admitted with fever tested positive to the DENV NS1 rapid test [39]. Based on a recent multicenter observational cohort study, the number of severe dengue cases in Indonesia is as low as 2.3\% [40]. The small number of participants in our study thus limits the occurrences of severe dengue cases. In addition, possible selection bias might have impacted both the numbers shown in these studies and the numbers that we report, because the design of the study meant that we had to rely on less sensitive, point-of-care testing.

In several studies, the complex role played by NS1 in cytokine release, endothelial disturbances and complement activation is discussed, as well as the both protective and detrimental effects attributed to NS1-specific antibodies during a secondary disease episode [41]. As this was not part of our objectives and given the small numbers in our study, no conclusions have been drawn from NS1 positivity and its relation to disease stage (i.e. the tendency, or otherwise, of developing bleeding complications) versus MultiPlate results. Clearly, the "fever of another origin" group is both anamnestic as well as biologically different, as is characterised, for instance, by a thrombocytosis and leukocytosis. As the authors are not acquainted with any other research on viral cases and usage of MultiPlate, besides the studies of Laine and colleagues [24] on Puumala orthohantavirus patients, it should be considered whether the significantly lower AUC values of ADP, COL and TRAP in DENV cases are virus-specific or whether they can be attributed to viral (haemorrhagic fever) infections in general. Given the extent to which thrombopathy is a hallmark in DENV infections, it is likely to fit with only a small number of viral infections, orthohantavirus and DENV included. In any case, from our data it would seem that a clear clinical suspicion of DENV infection (i.e. by first applying WHO 2009 clinical decision rules) and low AUC ADP values on hospital admission relates to a prolonged hospital stay.

The exact route by which DENV induces thrombopathy, of which ADP, COL and TRAP values measured by MultiPlate are a representation needs further study. In recent studies, for instance, the modulation of DC-SIGN and FcYR2A receptor expression on thrombocytes, was suggested to have a protective effect because it prevents tissue damage due to thrombocyte aggregation [42]. While targets for ADP, TRAP and COL might still be active, our results could support such findings in a way that the ability to stimulate thrombocyte aggregation is still possible thru ADP, TRAP and COL, but the resulting cascade to aggregation is temporarily impaired Trends to a stable recovery of ADP, TRAP and COL induced platelet aggregation can be observed in the D1 data, as well as in the sparse data available from D7 +/$48 \mathrm{~h}$. Also, recent in-vitro studies have shown that there are morphological changes in thrombocytes during DENV infections with changes in angiogenic and inflammatory profiles. This could possibly support our findings of impaired coagulation when thrombocytes are externally stimulated by means of ADP, TRAP and COL. Future studies should investigate whether this for instance might be due to conformational changes in receptor binding sites [13]. 
Results reported in the MultiPlate are a reflection of the potency of thrombocytes to aggregate in a fixed amount of time. However, the results are also influenced by the absolute numbers of thrombocytes, as has been reported in previous studies [30, 43]. For this reason, the arbitrary thrombocyte count of at least $100 \times 10^{9} / \mathrm{L}$ was chosen. Furthermore, the use of medication that could potentially interfere with MultiPlate reliability was considered as an exclusion criterion. Surprisingly, we found a positive and significant correlation for thrombocyte counts in the fever of another origin group versus ADP AUC at baseline. We could not replicate this correlation for the low ADP AUC values that seem to be specific to the DENV group, which strengthens our findings. Also, in this regard no significant observations could be made for the whole study group (data not shown). It might therefore be likely that the low ADP values that we observed are DENV-specific for those with a thrombocyte count of $>100 \times 10^{9} / \mathrm{L}$ at baseline.

A significant proportion of the regions in which DENV is endemic comprises countries that are considered low- to middle-income and in which some of their healthcare systems are characterised by having limited resources, from both a diagnostic and financial point of view. In outbreak situations, in particular, when many people might be simultaneously in need of medical care, the number of patients presenting at a hospital can easily exceed its capacity. Fast, reliable and cost-effective diagnostics that, preferably, predict the need for admission and/or intensified follow-up, would thus be highly relevant in these settings. Once set-up and validated using blood of healthy controls, the MultiPlate offers a fast turnaround time of less than $10 \mathrm{~min}$ and at a cost that is comparable to routine haematology and biochemistry laboratory tests. The combination of decreased aggregation, leukopenia and clinical signs like headache for rapid DENV diagnosis should be further studied, but our results so far suggest a potential role of thrombocyte function tests. Its eventual complementary role to molecular or ELISA/rapid based DENV diagnostics would however need further study. For this, we suggest that future studies should include molecular techniques to detect acute DENV, be conducted in several continents and be complemented with molecular testing on other viral haemorrhagic fevers. This then does not only account for circulating DENV serotypes but also defines the validity of our results for other haemorrhagic fevers.

\section{Conclusion}

In this explorative study of measuring whole blood aggregation of thrombocytes using ADP, COL and TRAP, we have found potentially relevant insights in thrombocyte function in acute DENV. While future exploration is needed, such tests potentially serve as an additional marker in acute DENV. These findings should be further explored in more extensive cohort studies or in outbreaks where the number of patients presenting to a hospital with symptoms of DENV exceeds the available hospital capacity.

\section{For all patients, the treating physician was requested to score the following dengue signs/ symptoms (following the WHO dengue 2009 guidelines - see main text for reference)}

- Headache.

- Leukopenia.

- Muscle/joint pain.

- Nausea/vomiting.

- Rash.

- Retro-orbital pain.

- Swollen glands.

Firstly, all subjects were tested for the presence of DENV NS antigen with a DENV NS1 rapid test (Panbio ${ }^{\circ}$ Dengue Early Rapid) and DENV NS1 ELISA (Panbio ${ }^{\circ}$ Dengue Early ELISA dengue NS1) according to the manufacturer's specifications. Those that tested positive (i.e. above the manufacturer-specified and/or per calculated cut-off value according to the manufacturer's instructions) were classified as "Dengue confirmed".

Secondly, for other enrolled subjects, a differential diagnose list was made by the supervising infectious diseases specialist. Patients whose fever was probably attributable to a different cause, such as suspected pneumonia, urosepsis or typhoid fever, for example, were classified in the group "fever of another origin".

Those admitted with an unclear cause of fever, due to having less pronounced symptoms, for example, were evaluated in accordance with dengue signs/symptoms as mentioned previously and, if accompanied by two or more positive signs/symptoms, were classified as "probable dengue".

Patients in which DENV infection was more than likely, but who were negative for NS1 ELISA, were evaluated in accordance with dengue signs/symptoms and, if accompanied by two or more positive symptoms were classified as "probable dengue".

\section{Supplementary information}

Supplementary information accompanies this paper at https://doi.org/10. 1186/s12879-020-05248-4.

Additional file 1: Table S1. Performance of NS1 rapid antigen test vs NS1 ELISA capture assay.

\section{Abbreviations}

DENV: Dengue virus; NS1: Non-structural protein 1; ELISA: Enzyme-linked immunosorbent assay; ADP: Adenosine diphosphate; TRAP-6: Thrombin 
receptor activator for peptide 6; COL: Collagen; AUC: Area Under the aggregation Curve; HC: Healthy controls; HFRS: Haemorrhagic Fever and Renal Syndrome; AU: Aggregation units; NSAIDs: Nonsteroidal antiinflammatory drugs

\section{Acknowledgements}

The authors would like to thank and acknowledge: all the patients who voluntarily participated; Dr. Abdullah Machin, Sp. S for his help in setting up the collaboration with the hospital; Prof. Dr. Nasronudin, Dr., Sp.PD-KPTI., FINASIM as director of the hospital; the nurses of the internal medicine department for their close involvement; Miss Nurul for her continuous support in the laboratory; Maria Arni Stella for collecting clinical data and processing samples; and Paul Verhezen for his advice on using the collagen reagent.

\section{Authors' contributions}

Conceptualization, W.J., M.R., E.G. U.H. and M.G.; Data curation, W.J. and I.V.; Formal analysis, W.J. and I.V.; Investigation, W.J., T.A., I.V.; Methodology, W.J., T.A., U.H. E.G. and M.G.; Project administration, T.A., I.V. and U.H.; Resources, M.R. and U.H.; Supervision, U.H., E.G. and M.G.; Validation, M.G.; Writing original draft, W.J. and M.G.; Writing - review \& editing, W.J., T.A., I.V., M.R., U.H., E.G. and M.G. The author(s) read and approved the final manuscript.

\section{Funding}

This research received no external funding.

\section{Availability of data and materials}

Data is available upon reasonable request to the corresponding author. Supplementary data is available from Appendix A and Table S1: Performance of NS1 rapid antigen test vs NS1 ELISA capture assay.

\section{Ethics approval and consent to participate}

Ethical clearance was obtained from the ethical committee of the Universitas Airlangga hospital, reference 126/KEH/2017. All study procedures were conducted following the Declaration of Helsinki. Written informed consent was obtained from all participants or their legal representatives(parents/ guardians of the minors included in this study_.

\section{Consent for publication}

Not applicable.

\section{Competing interests}

The authors report no competing interest.

\section{Author details}

'Department of Viroscience, Erasmus MC, Rotterdam, the Netherlands. ${ }^{2}$ Department of Internal Medicine, Universitas Airlangga Hospital, Airlangga University, Surabaya, Indonesia. ${ }^{3}$ Department of infectious diseases, Rumah Sakit Umum Daerah Dr Soetomo, Airlangga University, Surabaya, Indonesia. ${ }^{4}$ Department of internal medicine, Erasmus MC, Rotterdam, the Netherlands.

\section{Received: 21 November 2019 Accepted: 14 July 2020}

Published online: 06 August 2020

\section{References}

1. WHO. Dengue and severe dengue: World Health Organization; 2019. Updated 15-04-2019. Available from: https://www.who.int/news-room/factsheets/detail/dengue-and-severe-dengue.

2. Indonesia: The World Bank; 2019. Available from: https://data.worldbank.org/ country/indonesia.

3. de Jong W, Rusli M, Bhoelan S, Rohde S, Rantam FA, Noeryoto PA, et al. Endemic and emerging acute virus infections in Indonesia: an overview of the past decade and implications for the future. Crit Rev Microbiol. 2018; 44(4):487-503.

4. Wills BA, Nguyen MD, Ha TL, Dong TH, Tran TN, Le TT, et al. Comparison of three fluid solutions for resuscitation in dengue shock syndrome. N Engl J Med. 2005:353(9):877-89.

5. Nadjib M, Setiawan E, Putri S, Nealon J, Beucher S, Hadinegoro SR, et al. Economic burden of dengue in Indonesia. PLoS Negl Trop Dis. 2019;13(1): e0007038.
6. Shepard DS, Undurraga EA, Halasa YA. Economic and disease burden of dengue in Southeast Asia. PLoS Negl Trop Dis. 2013;7(2):e2055.

7. Phuong NTN, Manh DH, Dumre SP, Mizukami S, Weiss LN, Van Thuong N, et al. Plasma cell-free DNA: a potential biomarker for early prediction of severe dengue. Ann Clin Microbiol Antimicrob. 2019;18(1):10.

8. Davi CCM, Pastor A, Oliveira T, Lima Neto FB, Braga-Neto U, Bigham A, et al. Severe Dengue Prognosis Using Human Genome Data and Machine Learning. IEEE Trans Biomed Eng. 2019;66(10):2861-8.

9. Robinson M, Sweeney TE, Barouch-Bentov R, Sahoo MK, Kalesinskas L, Vallania F, et al. A 20-gene set predictive of progression to severe dengue. Cell Rep. 2019;26(5):1104-11 e4.

10. Low GK, Looi SY, Yong MH, Sharma D. Predictive and diagnostic test accuracy of ultrasonography in differentiating severe dengue from nonsevere dengue. J Vector Borne Dis. 2018;55(2):79-88.

11. Kotepui M, PhunPhuech B, Phiwklam N, Uthaisar K. Differentiating between dengue fever and malaria using hematological parameters in endemic areas of Thailand. Infect Dis Poverty. 2017;6(1):27.

12. Assinger A. Platelets and infection - an emerging role of platelets in viral infection. Front Immunol. 2014;5:649.

13. Barros T, Batista DO, Torrentes de Carvalho A, NRD CF, Barreto-Vieira DF, Jacome FC, et al. Different aspects of platelet evaluation in dengue: measurement of circulating mediators, ability to interact with the virus, the degree of activation and quantification of intraplatelet protein content. Virus Res. 2019;260:163-72.

14. Hapsari Putri I, Tunjungputri RN, De Groot PG, van der Ven AJ, de Mast Q. Thrombocytopenia and platelet dysfunction in acute tropical infectious diseases. Semin Thromb Hemost. 2018;44(7):683-90.

15. Hottz ED, Bozza FA, Bozza PT. Platelets in Immune Response to Virus and Immunopathology of Viral Infections. Front Med (Lausanne). 2018;5:121.

16. Guo L, Rondina MT. The era of Thromboinflammation: platelets are dynamic sensors and effector cells during infectious diseases. Front Immunol. 2019; 10:2204.

17. Hottz ED, Medeiros-de-Moraes IM, Vieira-de-Abreu A, de Assis EF, Vals-deSouza R, Castro-Faria-Neto HC, et al. Platelet activation and apoptosis modulate monocyte inflammatory responses in dengue. J Immunol. 2014; 193(4):1864-72.

18. Ojha A, Nandi D, Batra H, Singhal R, Annarapu GK, Bhattacharyya S, et al. Platelet activation determines the severity of thrombocytopenia in dengue infection. Sci Rep. 2017;7:41697.

19. Dhanoa A, Rajasekaram G, Hassan SS, Ramadas A, Azreen Adnan NA, Lau CF, et al. Risk factors and clinical outcome of profound thrombocytopenia in adult patients with DENV infections. Platelets. 2017;28(7):724-7.

20. Polzin A, Helten C, Dannenberg L, Mourikis P, Naguib D, Achilles A, et al. Platelet reactivity in patients on aspirin and Clopidogrel therapy measured by a new bedside whole-blood assay. J Cardiovasc Pharmacol. 2019;73(1): 40-7

21. Mannu GS, Macartney A, Lambert JR, Bettencourt-Silva JH, Lawn M, Lyall H, et al. The clinical utility of multiplate analyser measurement in platelet function testing following stroke and transient ischaemic attack. Eur J Haematol. 2015;94(2):138-44.

22. Cowman J, Mullers S, Dunne E, Ralph A, Ricco AJ, Malone FD, et al. Platelet behaviour on von Willebrand factor changes in pregnancy: consequences of haemodilution and intrinsic changes in platelet function. Sci Rep. 2017; 7(1):6354.

23. Akinosoglou K, Theodoraki S, Xanthopoulou I, Perperis A, Gkavogianni T, Pistiki A, et al. Platelet reactivity in sepsis syndrome: results from the PRESS study. Eur J Clin Microbiol Infect Dis. 2017;36(12):2503-12.

24. Laine $\mathrm{O}$, Joutsi-Korhonen L, Lassila R, Koski T, Huhtala H, Vaheri A, et al. Hantavirus infection-induced thrombocytopenia triggers increased production but associates with impaired aggregation of platelets except for collagen. Thromb Res. 2015;136(6):1126-32.

25. Puri RN, Colman RW. ADP-induced platelet activation. Crit Rev Biochem Mol Biol. 1997;32(6):437-502.

26. Manon-Jensen T, Kjeld NG, Karsdal MA. Collagen-mediated hemostasis. J Thromb Haemost. 2016;14(3):438-48.

27. Davi G, Patrono C. Platelet activation and atherothrombosis. N Engl J Med. 2007;357(24):2482-94.

28. WHO. Dengue. 2009. Available from: https://www.who.int/tdr/publications/ documents/dengue-diagnosis.pdf.

29. Horstick O, Jaenisch T, Martinez E, Kroeger A, See LLC, Farrar J, et al. Comparing the usefulness of the 1997 and 2009 WHO dengue case 
classification: a systematic literature review. Am J Trop Med Hyg. 2014;91(3): 621-34.

30. Peerschke El, Castellone DD, Stroobants AK, Francis J. Reference range determination for whole-blood platelet aggregation using the multiplate analyzer. Am J Clin Pathol. 2014;142(5):647-56.

31. Association WM. Declaration of Helsinki: World Medical Association; 2013.

32. Muller DA, Young PR. The flavivirus NS1 protein: molecular and structural biology, immunology, role in pathogenesis and application as a diagnostic biomarker. Antivir Res. 2013;98(2):192-208.

33. Duong V, Ly S, Lorn Try P, Tuiskunen A, Ong S, Chroeung N, et al. Clinical and virological factors influencing the performance of a NS1 antigencapture assay and potential use as a marker of dengue disease severity. PLoS Negl Trop Dis. 2011;5(7):e1244

34. Lima Mda R, Nogueira RM, Schatzmayr HG, dos Santos FB. Comparison of three commercially available dengue NS1 antigen capture assays for acute diagnosis of dengue in Brazil. PLoS Negl Trop Dis. 2010;4(7):e738.

35. Kaushik A, Yndart A, Kumar S, Jayant RD, Vashist A, Brown AN, et al. A sensitive electrochemical immunosensor for label-free detection of Zikavirus protein. Sci Rep. 2018;8(1):9700.

36. Hunsperger EA, Sharp TM, Lalita P, Tikomaidraubuta K, Cardoso YR, Naivalu $T$, et al. Use of a rapid test for diagnosis of dengue during suspected dengue outbreaks in resource-limited regions. J Clin Microbiol. 2016;54(8): 2090-5.

37. Dewi BE, Naiggolan L, Putri $\mathrm{DH}$, Rachmayanti $\mathrm{N}$, Albar S, Indriastuti NT, et al. Characterization of dengue virus serotype 4 infection in Jakarta, Indonesia. S Asian J Trop Med Public Health. 2014;45(1):53-61.

38. Sasmono RT, Wahid I, Trimarsanto H, Yohan B, Wahyuni S, Hertanto M, et al. Genomic analysis and growth characteristic of dengue viruses from Makassar. Indonesia. Infect Genet Evol. 2015;32:165-77.

39. Wardhani P, Aryati A, Yohan B, Trimarsanto H, Setianingsih TY, Puspitasari D, et al. Clinical and virological characteristics of dengue in Surabaya. Indonesia. PLoS One. 2017;12(6):e0178443.

40. Utama IMS, Lukman N, Sukmawati DD, Alisjahbana B, Alam A, Murniati D, et al. Dengue viral infection in Indonesia: epidemiology, diagnostic challenges, and mutations from an observational cohort study. PLoS Negl Trop Dis. 2019;13(10):e0007785.

41. Chen HR, Lai YC, Yeh TM. Dengue virus non-structural protein 1: a pathogenic factor, therapeutic target, and vaccine candidate. J Biomed Sci. 2018;25(1):58.

42. Tomo S, Mohan S, Ramachandrappa VS, Samadanam DM, Suresh S, Pillai AB, et al. Dynamic modulation of DC-SIGN and FcUpsilonR2A receptors expression on platelets in dengue. PLoS One. 2018;13(11):e0206346.

43. Moenen F, Vries MJA, Nelemans PJ, van Rooy KJM, Vranken J, Verhezen PWM, et al. Screening for platelet function disorders with multiplate and platelet function analyzer. Platelets. 2019;30(1):81-7.

\section{Publisher's Note}

Springer Nature remains neutral with regard to jurisdictional claims in published maps and institutional affiliations.

Ready to submit your research? Choose BMC and benefit from:

- fast, convenient online submission

- thorough peer review by experienced researchers in your field

- rapid publication on acceptance

- support for research data, including large and complex data types

- gold Open Access which fosters wider collaboration and increased citations

- maximum visibility for your research: over $100 \mathrm{M}$ website views per year

At BMC, research is always in progress.

Learn more biomedcentral.com/submissions 